\title{
The dynamics of the observed solar granulation: spatio-temporal variations of line of sight velocity and thermodynamic parameters
}

\author{
O. A. Baran* \\ Astronomical Observatory, Ivan Franko National University of Lviv, Kyryla i Mefodia str., 8, 79005, Lviv, Ukraine

\begin{abstract}
We investigated the dynamics of solar granulation, using the neutral iron line $\lambda \approx 639.3 \mathrm{~nm}$ profiles from highspatial resolution observations around the centre of the solar disc, in the non-perturbed region. We reproduced spatio-temporal variations of kinematic and thermodynamic parameters of solar convection at different heights of the solar photosphere $(h=-25 . .550 \mathrm{~km})$. The acoustic waves were removed by $k-\omega$ filtration. We analysed the temporal changes of these variations within convective cells.
\end{abstract}

Key words: solar granulation, line of sight velocity, thermodynamic parameters

\section{INTRODUCTION}

The most prominent intensity variations on the solar surface, aside from sunspots and faculae, are granules - the bright locations of upflowing hot plasma surrounded by dark lanes with downflowing cool plasma at scales of approximately $0.5-2 \mathrm{Mm}$ $[6,9]$. The organization and evolution of the granular cells in the solar photosphere reflect the physics of the upper convective layers, and have an effect on the higher layers, transferring energy, and momentum [4].

Over the past several years, significant efforts have been made to determine the stratification of physical quantities throughout the solar photosphere and to investigate the vertical structure of granulation. The classical picture of the convective breaks in the solar photosphere, starting at least from $50 \mathrm{~km}$ above the continuum formation level [3]. The higher layers of the photosphere are dominated by a type of secondary features, which are induced by overshooting granules. According to correlation analyses [1], the horizontal temperature fluctuations associated with these motions decrease rapidly with increasing height until they vanish. Only the largest granules $\left(>1^{\prime \prime} .5\right)$ contribute to the brightness pattern observed above where the brightness contrast is inverted. Note that the values of the height where the temperature fluctuations vanish, varies considerably throughout literature $[1,2,3,8,11]$. The values may differ from one another due to differences in the method used to establish the geometrical height scale (transformation from $\tau$ to $z$ ) and in different methods to filter the oscillations [8]. In [11] the authors

\footnotetext{
*lesiaab@gmail.com

(C) O.A.Baran, 2014
}

came to the conclusion that the temperature inversion occurs throughout a wide range of heights, from 150 to $500 \mathrm{~km}$. Additionally, the larger the granule, the higher the temperature inversion.

In [8] an average granular cell model is presented, showing the granule-intergranular lane stratification of temperature, vertical velocity, gas pressure and density as a function of height. The height variation of these physical quantities for structures of different size is depicted. The temperature inversion is found for structures with sizes over $1^{\prime \prime} .5$ at heights above $170 \mathrm{~km}$. The authors also report that only the large-scale structures (approximately $4^{\prime \prime} .0$ ) penetrate to higher layers without the loss of correlation though the entire atmosphere. Similar results were also reported in [7], where the authors find that only the intensity structures with sizes over $2^{\prime \prime} .0$ at heights about $435 \mathrm{~km}$ are still connected with those at the continuum level.

An important aspect of granular overshooting is the change of the convective velocity with height within the stable layers lying above [5]. In [1, 8] the vertical convective velocities were observed inphase though the entire photosphere penetrating into the highest layers being observed. The evidence of the vertical velocity reversal was first detected in [2] at heights above $490 \mathrm{~km}$ for the elements with the highest contrast in their data (above 6-9\%). In [3] the authors show that the heights of intensity contrast sign reversal and velocity sign reversal depend strongly on the granulation velocity and intensity at the bottom photosphere. This process occurs in a wide range of heights up to $650 \mathrm{~km}$.

In our work we represent the kinematic and ther- 
modynamic parameters of solar convection (vertical velocity, temperature, density and pressure) using profiles with high spatial resolution. We analyse their spatial and temporal changes to better understand the development process of convective cells. The aim of the present work is to investigate the vertical velocity stratification within granular structures as a function of height, to obtain evidence of the velocity reversal and to show the temporal changes of temperature, density and pressure of variations within such structures during their evolution.

\section{OBSERVATIONS AND DATA PROCESSING}

The data used for the study consists of a time sequence (947 images, observation duration $2.6 \mathrm{~h}$ ) of 512 profiles of the neutral iron spectral line with $\lambda \approx 639.3 \mathrm{~nm}$ taken by N. Shchukina in August 2001 at the 70 -cm German Vacuum Tower Telescope (VTT) located on the Canary Islands (Spain) [2]. The observation target location was within the solar disc centre, in the non-perturbed region. The image tremor on the input slit of the spectrograph did not exceed 0.5 arcsec, so the spatial resolution was equal to $350 \mathrm{~km}$. The data characterizes the region extending up to $64,400 \mathrm{~km}$ over the surface of the Sun. The region of line formation extends from several kilometres up to $550 \mathrm{~km}$ in height.

We reproduced line-of-sight velocities and variations of temperature, density, and pressure in the solar photosphere, using the sophisticated technology developed by Stodilka [10], who solved the inverse radiative transfer problem using modified response functions and Tikhonov's stabilisers. The acoustic waves were removed through $k-\omega$ filtration.

\section{RESULTS AND DISCUSSION}

For better visualisation we chose an area with a width of $X \approx 15 \mathrm{Mm}$ (i.e. $\approx \frac{1}{4}$ of the observable region) to show the typical changes of the reproduced variations that occur within the convective cells (Fig. 1-4).

\section{VERTICAL VELOCITY STRATIFICATION WITHIN CONVECTIVE CELLS}

Fig. 1 shows the velocity field at this area during the time interval $\Delta t_{a . . d} \approx 10 \mathrm{~min}$. The white colour corresponds to the velocity of the ascending flows of the matter, and the black colour-to that of the descending ones. The range of vertical velocity variations is constrained by $\pm 0.35 \mathrm{~km} / \mathrm{s}$ in order to obtain a better contrast in the upper layers of the atmosphere.

In the solar photosphere (see Fig. 1) the vertical velocity field has a columnar structure: the velocities are negative (descending flows) within granules and positive (ascending flows) within intergranules. The most common examples of the evolution of convective flow are presented at $X \approx 36.9 \mathrm{Mm}$ and at
$X \approx 44 \mathrm{Mm}:$ the descending flows stem from the lower photosphere (Fig. 1a-b), then these flows penetrate from the lower layers of the photosphere into the upper layers (Fig. 1b-c), until they reach the maximal values of vertical velocities (Fig.1c) and vanish (Fig. 1c).

In some cases the vertical velocities change sign in the upper photosphere, so matter starts moving downward within such granules and upward within intergranules - the inversion of velocities takes place. Thus, in Fig. 1a the velocity inversion is found at $X=43.0 . .43 .8 \mathrm{Mm}$ in two neighbouring ascending flows that are located close to each other (there is a thin descending flow between them). The wide and strong ascending flow originates at $X \approx 33.2 \mathrm{Mm}$ in Fig. $1 \mathrm{~b}$, and at the same time, the opposite flow arises from the top, i. e. the matter moves up in the lower photosphere and down at the heights $h>250 \mathrm{~km}$; in a time period of $\Delta t_{b . . c} \approx 3 \mathrm{~min}$, the ascending flow increases and the height of the inversion is elevated. Eventually the opposite (descending) flow disappears in the upper layers, while the ascending flow reaches the upper photosphere (Fig. 1c) and then descends (see Fig. $1 d$ after $\Delta t_{c . . d} \approx 3 \mathrm{~min}$ ). The weak ascending flow resides in the lower layers of the photosphere at $X \approx 44.8 \mathrm{Mm}$ in Fig. 1b along with descending flows, apart from where they merge together in the upper layers. After a while (see Fig. 1c) this ascending flow is destroyed without reaching the upper photosphere. Similarly, the velocity inversion occurs within the descending flow at $X \approx 34.2 \mathrm{Mm}$ and at $X \approx 36.1 \mathrm{Mm}$ at the end of their existence in Fig. 1a-b. After the time interval $\Delta t_{b . . c} \approx 3 \mathrm{~min}$ (see Fig. 1c) the first flow disappears and the descending flow develops in its place; the second flow decreases and merges with the neighbouring flow, and the inversion vanishes.

The present data shows the various cases of motion of the matter within convective cells. The velocity reversal was found, which is in agreement with the results obtained in [3]. However, for all the observed time throughout the reproduced region the inversion of vertical velocities is less common, at least at the heights $h=-25 . .550 \mathrm{~km}$ that we consider (in [3] the velocity sign reversal is possible over the whole atmosphere from 50 to $650 \mathrm{~km}$ ). In our case, the velocity inversion occurs within $12 \%$ of the convective cells during their evolution at the heights from about $h \approx 200 \mathrm{~km}$ and higher (up to the heights of temperature minimum).

\section{THE VARIATIONS OF THERMODYNAMIC \\ PARAMETERS WITHIN CONVECTIVE CELLS}

Now consider how the variations of temperature (Fig. 2), pressure (Fig. 3) and density (Fig. 4) occur at the same area and in the same time interval. In the solar photosphere the pressure and density vary strongly with heights, so we examine their relative variations to get a better indication of these vari- 
ations in the upper photosphere. In order to gain better contrast, the ranges of the variations are constrained by $\pm 125 \mathrm{~K}$ for temperature, \pm 0.06 for the relative variations of pressure and \pm 0.06 for the relative variations of density: light shading depicts positive variations and dark shading depicts negative ones.

In the lower photosphere the temperature variations (see Fig. 2) are positive within ascending flows and negative within descending flows, and they decrease above. At the heights $h>200 \mathrm{~km}$ the temperature variations reach minimum and then increases again but with opposite sign: a temperature inversion takes place. Our results are similar to those obtained in [8] $(h \approx 170 \mathrm{~km})$ and are in agreement with the results in [3] $(h \approx 200-300 \mathrm{~km})$.

In Fig. 3 the relative variations of pressure are presented. These variations are minimal in the lower photosphere and increase with height: they become positive within granules and negative within intergranules. The received stratification of the relative variations of pressure is consistent with that obtained in [8].

The relative variations of density are shown in Fig. 4. They are minimal in the lower photosphere: negative variations are found within ascending flows and positive variations - within descending flows. In the layers $h \approx 75 \mathrm{~km}$ a density reversal occurs. Above this height the relative variations of density increase: they become positive within granules and negative within intergranules. The received stratification of these variations is close to that obtained in [8]. However, the authors found the inversion of the relative variations of density somewhat lower - at the heights $h \approx 30 \mathrm{~km}$.

\section{THE EVOLUTION OF CONVECTIVE FLOWS}

In Fig. 1a-b we saw the evolution of vertical velocities in the descending flows at $X \approx 36.9 \mathrm{Mm}$ and at $X \approx 44.0 \mathrm{Mm}$. At the same time their temperature structures (see Fig. 2) were formed: the temperature variations increase within these cells in the lower photosphere and the opposite variations increase above them at the heights $h>200 \mathrm{~km}$. At $X \approx 36.9 \mathrm{Mm}$ the temperature structure develops faster than the flow (see Fig. 1b-c and Fig. 2b-c) and the temperature inversion occurs from the beginning. At $X \approx 44.0 \mathrm{Mm}$ the temperature inversion is found when the flow is well-developed (see Fig. 1c and Fig. 2c). The relative variations of density (Fig. 3) and pressure (Fig. 4) within these structures increase to the maximal values in the upper layers of the photosphere and decrease when the flows vanish.

If velocity inversion occurs at the beginning of the flow development, but disappears quickly, the flow increases and reaches the higher layers. An example of such development is the flow at $X \approx 33.2 \mathrm{Mm}$ in Fig. 1b-d. In this case, the temperature inversion appears at heights $h>375 \mathrm{~km}$ (Fig. 2c). In the upper photosphere the relative variations of density and pressure increase within the flow as well, but with some delay (see $X \approx 33.2 \mathrm{Mm}$ in Fig. 3b-d and Fig. 4b-d).

If the velocity inversion prevents normal development of the flow (oppositely directed flow from above is fast and wide enough, so it leads to slow down the weak flow at the bottom (at $X \approx 44.8 \mathrm{Mm}$ in Fig. $1 \mathrm{~b}-\mathrm{c})$ ), the temperature structure disintegrates relatively rapidly (see Fig. $2 \mathrm{~b}-\mathrm{c}$ ). In such a case, the relative variations of density and pressure appear and disappear within a shot period of time (Fig. 3b-c and Fig. 4b-c).

\section{CONCLUSIONS}

We analysed the vertical velocities, the temperature variations and the relative variations of density and pressure in the solar photosphere using the neutral iron line $\lambda \approx 639.3 \mathrm{~nm}$ profiles from the observations with high spatial resolution taken around the centre of the solar disc in the non-perturbed region. The acoustic waves were removed through $k-\omega$ filtration. Our basic results can be summarised as follows:

- The vertical flows begin in the lower layers of the photosphere and then penetrate into the upper layers; the velocity inversion occurs at the heights $h>200 \mathrm{~km}$ within $12 \%$ of the convective cells at various moments of their developments (at the initial stage or later).

- During the evolution of convective flows their temperature structures are formed and usually the temperature inversion is found at the heights $h>200 \mathrm{~km}$.

- In the lower photosphere the relative variations of density and pressure are minimal. At the beginning of flow development the relative variations of density and pressure increase in the upper layers of the photosphere and decrease when the flow disintegrates.

The received stratification of the kinematic and thermodynamic parameters is consistent with that obtained in [8]. However, in [8] the inversion of vertical velocities was not found. In our case the vertical velocities may change sign in the upper photosphere, in the same way as it was detected in [3].

\section{ACKNOWLEDGEMENT}

The authors are grateful to Dr. N. G. Shchukina for kindly proving the results of observations and to Dr. R. I. Kostik for data reduction.

\section{REFERENCES}

[1] Espagnet O., Muller R., Roudier T., Mein N. \& Mein P. 1995, A\&AS, 109, 79 
[2] Kostyk R. I. \& Shchukina N. G. 2004, Astron. Rep., 48, 769

[3] Kostik R., Khomenko E. \& Shchukina N. 2009, A\&A, 506,1405

[4] Muller, R. 1999. in 'Motions in the Solar Atmosphere', eds.: Hanslmeier A. \& Messerotti M., Kluwer Academic Publishers, Dordrecht, Boston, London, 239, 35

[5] Nesis A., Hammer R., Roth M. \& Schleicher H. 2006, A\&A, 451, 1081

[6] Nordlund Å., Stein R. F. \& Asplund M. 2009, Liv. Rev.
Sol. Phys., 6, 2

[7] Puschmann K., Vázquez M., Bonet J. A., Ruiz Cobo B. \& Hanslmeier A. 2003, A\&A, 408, 363

[8] Puschmann K. G., Ruiz Cobo B., Vázquez M., Bonet J. A. \& Hanslmeier A. 2005, A\&A, 441, 1157

[9] Rieutord M., Roudier T., Rincon F. et al. 2010, A\&A, $512, \mathrm{~A} 4$

[10] Stodilka M. 2002, J. Phys. Studies, 6, 435

[11] Stodilka M. I., Baran O. A. \& Malynych S. Z. 2006, Kinematika i Fizika Nebesnykh Tel, 22, 3, 173 
(a)

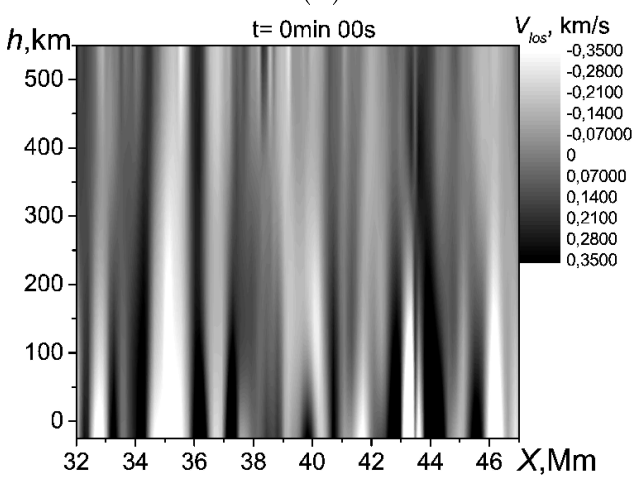

(b)

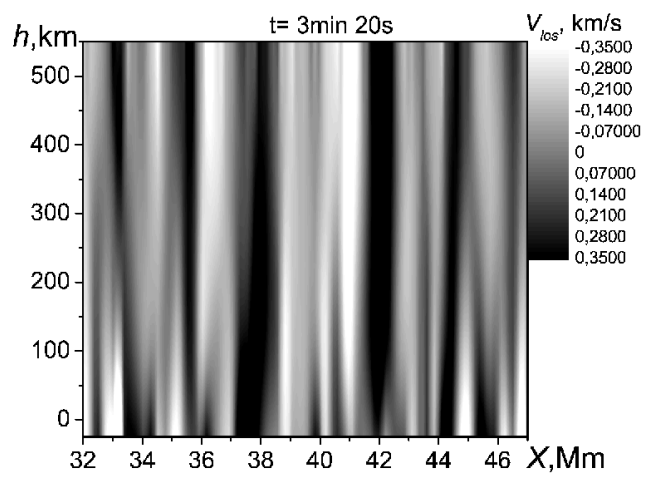

(c)

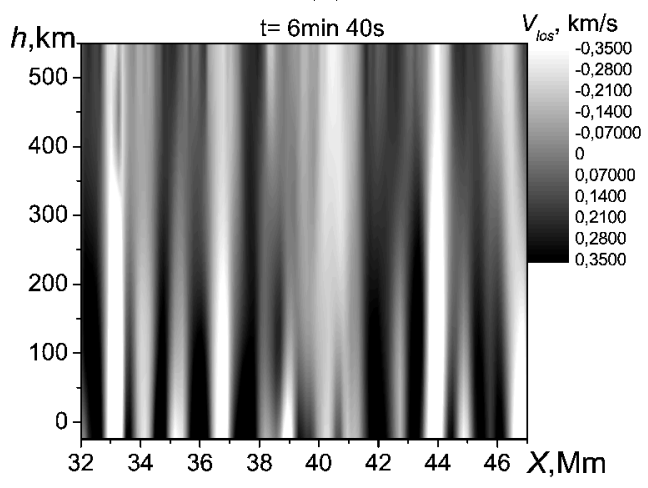

(d)

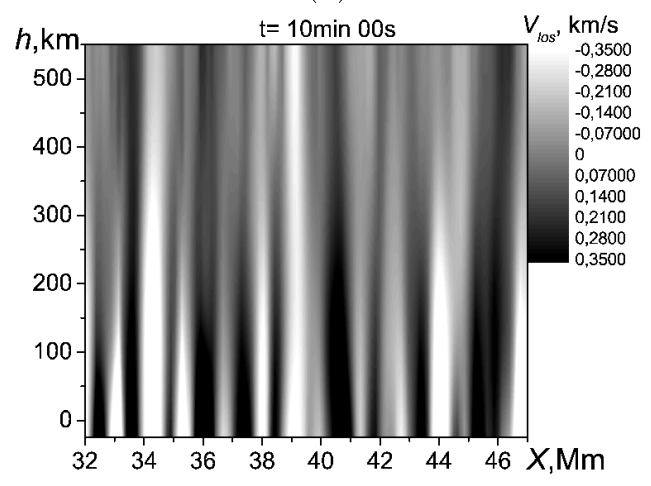

Fig. 1: The convective velocity stratification in the solar photosphere during $\Delta t_{a-d} \approx 10 \mathrm{~min}$. (a)

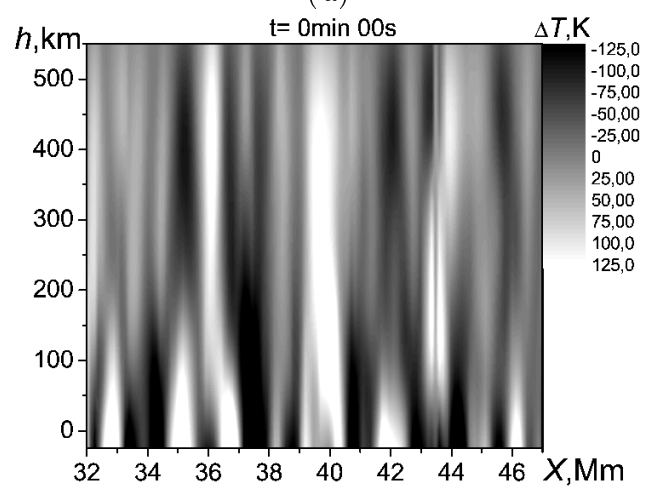

(b)

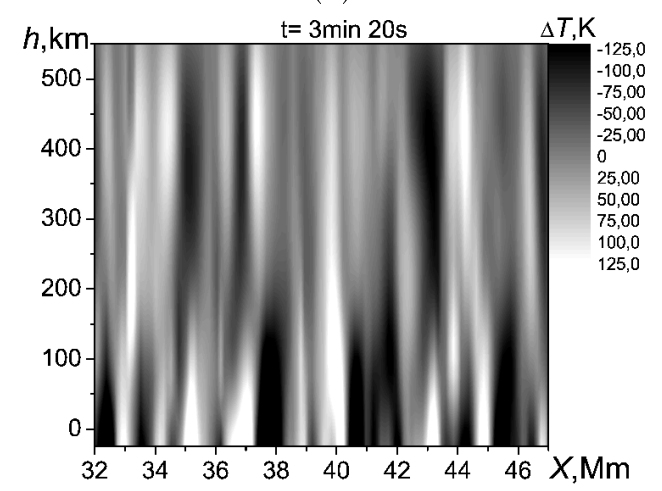

(c)

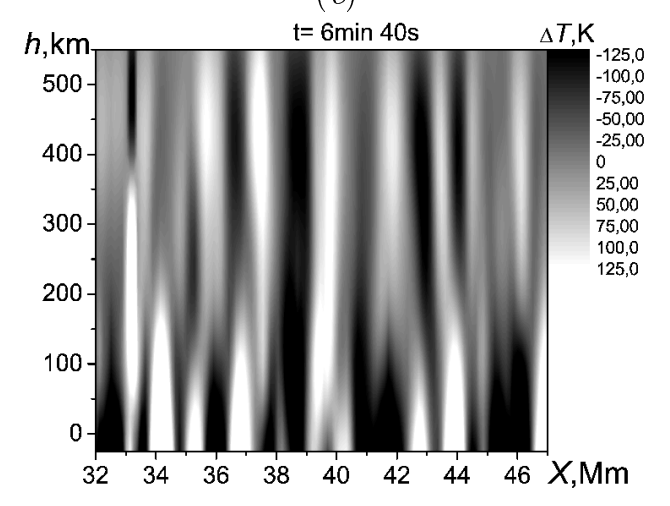

(d)

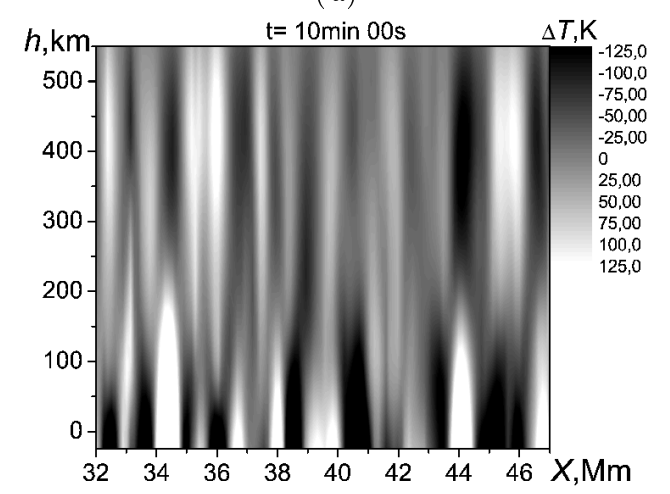

Fig. 2: The temperature variations of the photospheric convection at the same time as in Fig.1. 
(a)

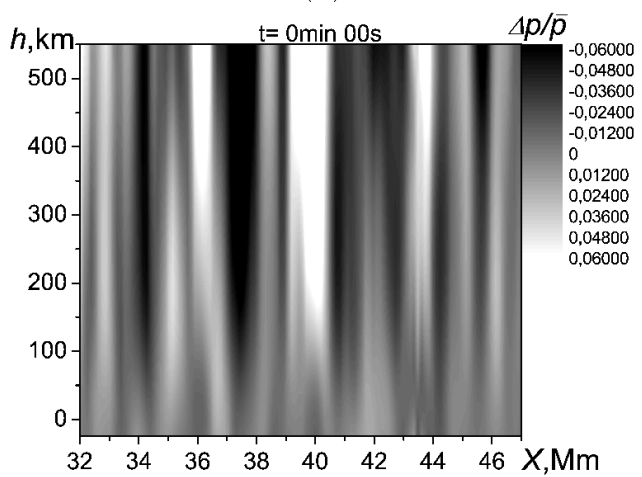

(b)

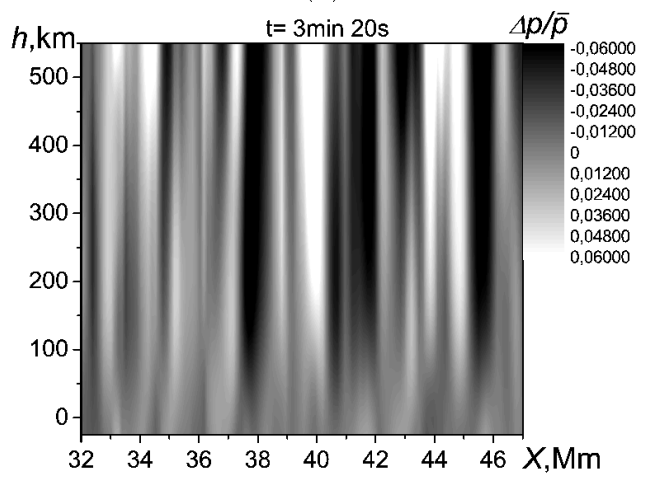

(c)

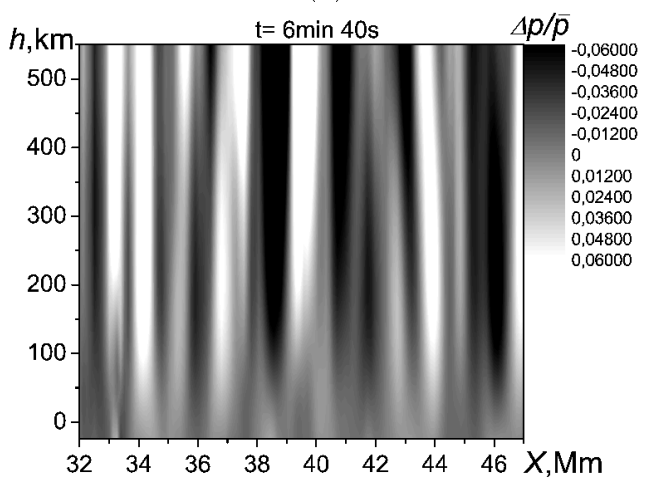

(d)

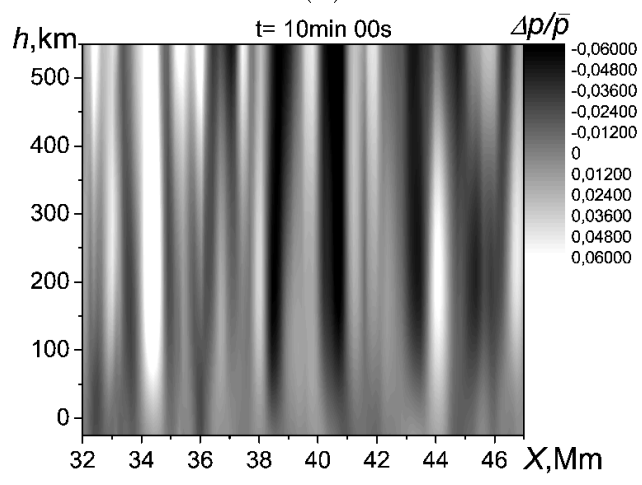

Fig. 3: The relative variations of pressure of the photospheric convection at the same time as in Fig.1. (a)

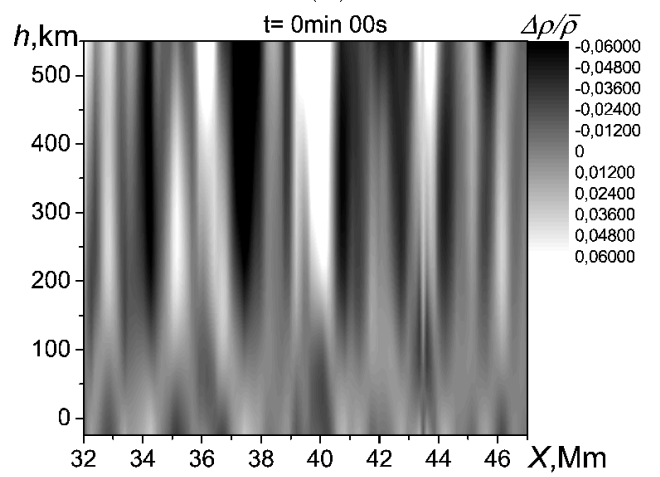

(b)

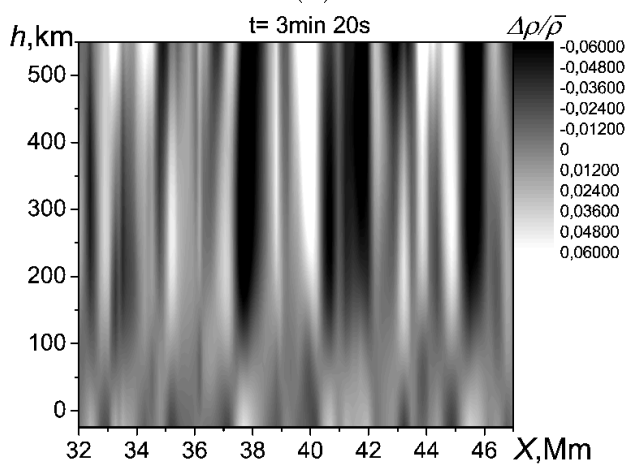

(c)

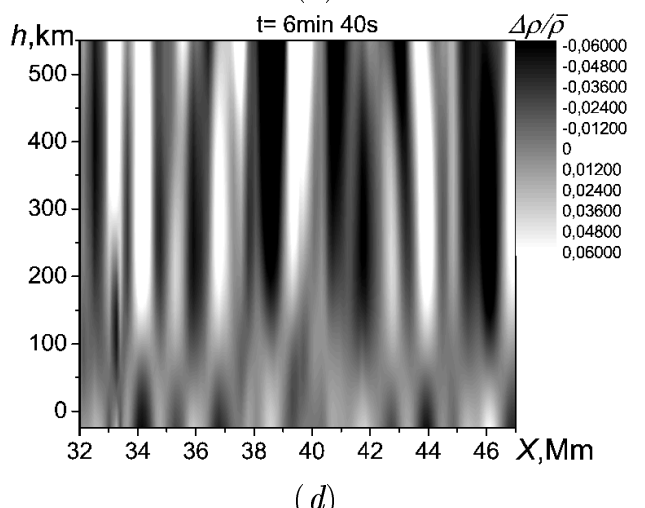

(d)

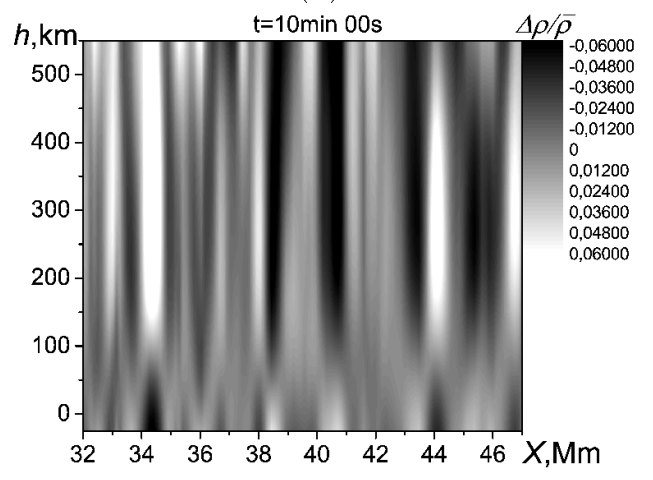

Fig. 4: The relative variations of density of the photospheric convection at the same time as in Fig.1. 